\title{
Lysophosphatidylcholine is a Major Component of Platelet Microvesicles Promoting Platelet Activation and Reporting Atherosclerotic Plaque Instability
}

\author{
Philipp Diehl ${ }^{1,2}$ Frederik Nienaber $^{1} \quad$ Maria T. K. Zaldivia ${ }^{1}$ Johannes Stamm ${ }^{1}$ Patrick M. Siegel ${ }^{1,2}$ \\ Natalie A. Mellett ${ }^{3}$ Marius Wessinger ${ }^{2}$ Xiaowei Wang ${ }^{1}$ James D. McFadyen ${ }^{1}$ Nicole Bassler ${ }^{1}$ \\ Gerhard Puetz ${ }^{4}$ Nay M. Htun ${ }^{1}$ David Braigi, ${ }^{1,5}$ Jonathon Habersberger ${ }^{1}$ Thomas Helbing ${ }^{2}$ \\ Steffen U. Eisenhardt ${ }^{5}$ Maria Fuller ${ }^{6}$ Christoph Bode ${ }^{2}$ Peter J. Meikle ${ }^{3}$ Yung Chih Chen ${ }^{1}$ \\ Karlheinz Peter ${ }^{1}$
}

1 Department of Atherothrombosis and Vascular Biology, Baker Heart and Diabetes Institute, Melbourne, Australia

2 Department of Cardiology and Angiology I, Faculty of Medicine, Heart Center - University of Freiburg, University of Freiburg, Freiburg, Germany

${ }_{3}^{3}$ Metabolomics Laboratory, Baker Heart and Diabetes Institute, Melbourne, Australia

${ }^{4}$ Institute for Clinical Chemistry and Laboratory Medicine, University of Freiburg, Freiburg, Germany

${ }^{5}$ Department of Plastic and Hand Surgery, University of Freiburg, Freiburg, Germany

${ }^{6}$ Centre for Molecular Pathology, University of Adelaide, Adelaide, Australia

\author{
Address for correspondence Karlheinz Peter, MD, PhD, Baker Heart \\ and Diabetes Institute, 75 Commercial Road, Melbourne, Victoria \\ 3004, Australia (e-mail: karlheinz.peter@baker.edu.au).
}

\begin{abstract}
Keywords

- lysophosphatidylcholine

- microvesicles

- platelet activation

- unstable atherosclerotic plaques

Background Microvesicles (MVs) are small cell-derived vesicles, which are mainly released by activated cells. They are part of a communication network delivering biomolecules, for example, inflammatory molecules, via the blood circulation to remote cells in the body. Platelet-derived MVs are known to induce vascular inflammation. Research on the mediators and mechanisms of their inflammatory effects has attracted major interest. We hypothesize that specific lipids are the mediators of vascular inflammation caused by platelet-derived MVs.

Methods and Results Liquid chromatography electrospray ionization-tandem mass spectrometry was used for lipid profiling of platelet-derived MVs. Lysophosphatidylcholine (LPC) was found to be a major component of platelet-derived MVs. Investigating the direct effects of LPC, we found that it induces platelet activation, spreading, migration and aggregation as well as formation of inflammatory platelet-monocyte aggregates. We show for the first time that platelets express the LPC receptor G2AR, which mediates LPC-induced platelet activation. In a mouse model of atherosclerotic plaque instability/rupture, circulating LPC was detected as a surrogate marker of plaque instability. These findings were confirmed by matrix-assisted laser desorption ionization imaging, which showed that the LPC concentration of human plaques was highest in vulnerable plaque regions.
\end{abstract}

received

September 16, 2018

accepted after revision January 25, 2019
DOI https://doi.org/

10.1055/s-0039-1683409.

ISSN 0340-6245. (c) 2019 Georg Thieme Verlag KG Stuttgart · New York
License terms

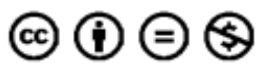


Conclusion LPC is a major component of platelet-derived MVs and via its interaction with G2AR on platelets contributes to platelet activation, spreading, migration and aggregation and ultimately to vascular inflammation. Circulating LPC reports on atherosclerotic plaque instability in mice and is significantly increased in unstable areas of atherosclerotic plaques in both mice and humans, linking LPC to plaque instability.

\section{Introduction}

Microvesicles (MVs), also called microparticles, are small cell blebs typically released during the activation and apoptosis of multiple cell types. ${ }^{1-3}$ They possess the surface characteristics of their parental cells; hence, their cells of origin can be identified. ${ }^{4}$ Even though it was initially believed that MVs are only cellular debris, their shedding into the blood is a highly regulated process. ${ }^{1,2}$ Once released from their parental cells into circulation, MVs bind in a receptor-mediated way to distinct target cells, transferring their cell membrane as well as their vesicle components to their destination cell. ${ }^{5,6}$ The biology of MVs is highly complex and only partly understood, but there is increasing evidence that they contain inflammatory ribonucleic acids (RNAs) and cytokines, which they deliver to their destination cells. ${ }^{7,8}$ Furthermore, MVs are 'loaded' by their parental cells with distinct RNA molecules, underlining the highly complex biology of MV release. ${ }^{6}$ The MV-dependent transfer of bioactive molecules often results in altered pro-inflammatory function and phenotype of the target cells, remote from the original source of these MVs, as demonstrated for endothelial cells, smooth muscle cells, monocytes, neutrophils and T cells. ${ }^{9-13}$ Hence, MVs are part of a unique communication network involved in a multitude of diseases. ${ }^{3,14,15}$ In particular, platelet-derived MVs (PMVs) have been associated with cardiovascular diseases. ${ }^{2,10}$

Lysophosphatidylcholine (LPC; 2-acyl-sn-glycero-3-phosphocholines) is a phospholipid generated from phosphatidylcholine (PC) after removal of a fatty acid group. LPC is increased in cardiovascular diseases driven by acute and chronic inflammation. ${ }^{16}$ As circulating, unbound LPC is immediately degraded by plasma lysophospholipases, it remains only temporarily in circulation if unprotected from degradation. However, MVs have been proposed to be transport vehicles for LPC. ${ }^{17,18}$

This study describes LPC as a major component of circulating PMVs and investigates its effects on platelet function and its potential link to atherosclerotic plaque instability.

\section{Methods}

\section{Platelet-Derived MV Preparation}

Blood was taken from the antecubital veins of healthy individuals and anticoagulated by citrate (Fc $0.01 \mathrm{M}$ ). Samples were centrifuged $(150 \times g, 15$ minutes $)$ to obtain platelet-rich plasma (PRP). To obtain highly pure PMV samples, platelets were washed using the following protocol. PRP was centri- fuged $(720 \times g$, 10 minutes, room temperature [RT]) and the platelet-containing pellets were re-suspended in acid citrate dextrose (ACD) Tyrode's buffer containing prostaglandin (1 $\mu \mathrm{M})$. After a second centrifugation step $(500 \times g, 10$ minutes, $\mathrm{RT}$ ), platelets were re-suspended in the initial volume of PRP with Tyrode's buffer containing $1 \mathrm{mM}$ of $\mathrm{MgCl}_{2}$ and $\mathrm{CaCl}_{2}$. MV release was induced by adding agonists (adenosine diphosphate [ADP] $20 \mu \mathrm{M}$, thrombin $0.1 \mathrm{U} / \mathrm{mL}$ and collagen $10 \mu \mathrm{g} / \mathrm{ml}$ ) for 15 minutes at RT. Platelets were pelleted by centrifugation $(16,000 \times g, 2$ minutes $)$ and MVs were purified by ultracentrifugation $\left(100,000 \times \mathrm{g}, 60\right.$ minutes, $\left.4^{\circ} \mathrm{C}\right)$, washed in phosphate-buffered saline (PBS) and subjected to lipid mass spectrometry. Significant contamination of PMV preparations by lipoproteins, which could carry LPC, has been excluded by lipoprotein analysis of the PMV samples.

\section{Lipid Analysis with Liquid Chromatography Electrospray Ionization-Tandem Mass Spectrometry}

Lipid extraction of MVs was performed as follows: Samples underwent total lipid extraction using a single-phase chloroform/methanol (2:1) technique, as described previously. ${ }^{19} \mathrm{~A}$ $10-\mu \mathrm{L}$ aliquot of cell lysate or MVs was combined with $200 \mu \mathrm{L}$ $\mathrm{CHCl} 3 / \mathrm{MeOH}(2: 1)$ and $15 \mu \mathrm{L}$ internal standard mix, then briefly vortexed. Samples were mixed (rotary mixer, 10 minutes), sonicated (water bath, 30 minutes) and allowed to stand (20 minutes) at RT. Samples were centrifuged $(16,000 \times g$, 10 minutes) and the supernatant dried under a stream of nitrogen $\left(\mathrm{N}_{2}\right)$ at $40^{\circ} \mathrm{C}$. The extracted lipids were re-suspended in $50 \mu \mathrm{L}$ water-saturated $\mathrm{BuOH}$ with sonication (10 minutes), followed by $50 \mu \mathrm{L}$ of $10 \mathrm{mM} \mathrm{NH}_{4} \mathrm{COOH}$ in $\mathrm{MeOH}$. Extracts were centrifuged $(3,350 \times g, 5$ minutes $)$ and the supernatant transferred into $0.2 \mathrm{~mL}$ glass vials with Teflon insert caps. Mass spectrometric analysis was performed using $5 \mu \mathrm{L}$ injections of the lipid extracts. Lipid analysis was performed by liquid chromatography electrospray ionization-tandem mass spectrometry (LC ESI-MS/MS) using an Agilent 1200 liquid chromatography system with an Applied Biosystems API 4000 Q/ TRAP mass spectrometer with a turbo ion-spray source $\left(350^{\circ}\right.$ C) and the Analyst 1.5 data system. Quantification of lipids was based on signal intensity relative to the corresponding internal standard, as described previously. ${ }^{19}$ For lyophilized samples, background values of buffer and media were subtracted. Values displayed for each lipid class were calculated as the sum of each individual species within the class.

For lipid analysis of vessel tissue, tissue was first homogenized in PBS. Note that $100 \mu \mathrm{L}$ of homogenized sample were incubated with $\mathrm{CHCl}_{3} / \mathrm{MeOH}(2: 1,20$ volumes, 10 minutes, 
RT), with shaking (90 revolutions per minute [rpm]), followed by a second incubation step ( 50 minutes, RT). Note that $0.4 \mathrm{~mL}$ of $\mathrm{MQ} \mathrm{H}_{2} \mathrm{O}$ was added to each sample followed by incubation on a platform shaker for 10 minutes at $90 \mathrm{rpm}$. Then, samples were centrifuged at 3,500 rpm for 5 minutes before the upper aqueous layer was discarded. The lower hydrophobic layer, which contains phospholipids, was transferred into a clean glass tube. The hydrophobic layer was dried under $\mathrm{N}_{2}$ at $40^{\circ} \mathrm{C}$. For mass spectrometry analysis, dried samples were re-suspended in water-saturated butanol $(50 \mu \mathrm{L})$ and $10 \mathrm{mM}$ ammonium formate in $\mathrm{MeOH}(50 \mu \mathrm{L})$. Lipidomic analysis was then performed by LC ESI-MS/MS as described above.

\section{Preparation of LPC and PC Liposomes}

Organic solvents (e.g. chloroform and methanol), in which polar lipids such as LPC and PC need to be re-suspended, can affect cell integrity. To avoid any artificial effect of the lipid solvent, experiments were performed with lipid micelles that can be re-suspended in PBS. ${ }^{20}$ Therefore, as recommended by the manufacturer, LPC and PC powder (both AVANTI, Alabama, United States) were diluted in chloroform:methanol (1:2) and dried under $\mathrm{N}_{2}$ before the solvent was eliminated under vacuum. PBS ( $3 \mathrm{~mL})$ was added to the dried lipid powder and lipids were re-suspended under ultrasound exposure (30 seconds, RT), incubated at $80^{\circ} \mathrm{C}$ (20 minutes) and samples again sonicated ( 30 seconds). Lipid micelles were stored at $4^{\circ} \mathrm{C}$ and sonicated for 30 seconds before use. The molarity of the lipids in the micelle preparations was determined by mass spectrometry.

\section{Cell Culture Assay}

To investigate whether the LPC receptor, G2A receptor (G2AR), is expressed on platelets and to avoid any contamination-related artificial effect on G2AR expression analysis, DAMI (ATCC, United States) cells were used. DAMI cells are a megakaryoblastic cell line showing several platelet characteristics and often used as a contamination-free model to study platelets. ${ }^{21}$ The human cell line HeLa (ATCC) was used as a negative control. G2AR expression was assessed by quantitative reverse transcription-polymerase chain reaction (qRT-PCR) as described below.

To obtain highly pure platelet samples for functional assays, washed platelets were used. PRP was centrifuged $(720 \times \mathrm{g}, 10$ minutes, RT) and the platelet-containing pellets were re-suspended in ACD Tyrode's buffer containing prostaglandin $(1 \mu \mathrm{M})$. After a second centrifugation step $(500 \times g, 10$ minutes, RT), platelets were re-suspended in the initial volume of PRP with Tyrode's buffer containing $\mathrm{MgCl}_{2}$ and $\mathrm{CaCl}_{2}$. Washed platelets were counted (Sysmex KX-21N, Germany), normalized and used within 60 minutes for further assays.

\section{Flow Cytometry}

The effects of LPCs on the cell surface expression of different receptors were assessed with flow cytometry. All flow cytometric measurements were performed with a FACSCanto (BD Bioscience, United States) and data were processed with a FACSDiva (BD Bioscience).
Platelet surface receptor expression was assessed in washed, highly pure platelets incubated with LPC (10 and 25 $\mu \mathrm{M})$, PC $(10$ and $25 \mu \mathrm{M})$ or ADP $(20 \mu \mathrm{M})\left(10\right.$ minutes, $\left.37^{\circ} \mathrm{C}\right)$, followed by anti-CD62P or procaspase-activating compound 1 incubation (both BD Biosciences, 10 minutes, RT) and fixation (cell FIX, BD Bioscience). For G2AR blockage, a commercial anti-G2AR antibody (Santa Cruz, United States) was used.

As an additional indicator of vascular inflammation, the effects of LPC and PC on the number of platelet-monocyte aggregates (PMAs) were investigated. Whole blood was haemolyzed (BD FACS Lysing Solution, United States) and the remaining leukocytes and platelets were incubated with LPC, PC or ADP $\left(10\right.$ minutes, $\left.37^{\circ} \mathrm{C}\right)$. Samples were stained with antiCD14 and anti-CD41 antibodies (both Beckman Coulter, United States) and PMAs were defined as CD41-positive events located within the flow cytometric gate for monocytes.

For the assessment of PMV surface characteristics, annexin $\mathrm{V}$ binding buffer ( $1 \times$ final solution; BD Bioscience) was added to PMVs. The samples were incubated for 15 minutes in the dark with AF647-conjugated Annexin V (Thermo Fisher, United States), anti-CD41, anti-CD61 and anti-CD42b antibodies. After incubation, $450 \mu \mathrm{L}$ of annexin $\mathrm{V}$ binding buffer $(1 \times$ final solution) was added to the samples.

\section{Immunofluorescence Microscopy of Permeabilized Platelets}

Purified platelets were re-suspended in PBS with $0.1 \%$ bovine serum albumin (BSA) plus $0.1 \%$ glucose and adjusted to $10 \times 10^{3} / \mu \mathrm{L}$. Platelets were incubated with LPC, PC, PBS or ADP $(20 \mu \mathrm{M})$ for 30 minutes at $37^{\circ} \mathrm{C}$ and fixed with $2 \%$ paraformaldehyde. Platelets were placed into 6-well plates, each well containing a coverslip (MENZEL-Glaeser, Germany) coated with $0.01 \%$ poly-l-lysine (Sigma Aldrich, Germany). To fix platelets on the coverslips, the 6-well plates were centrifuged $(250 \times g$, 5 minutes $)$ and the platelets subsequently permeabilized (0.5\% Triton X 100, Sigma Aldrich, 5 minutes) before the slides were blocked overnight. Specimens were then incubated with a phycoerythrin-labelled anti-CD62P antibody (BD Bioscience) for 2 hours followed by several washing steps. Microscopy was performed on a Zeiss 510 Meta Confocal Microscope with a $40 \times$ water lens and images were processed with ImageJ 1.46n (United States).

\section{Platelet Static Adhesion Assay}

To investigate LPC effects on platelet adhesion and spreading, platelet static adhesion assays on immobilized fibrinogen were performed. ${ }^{22}$ Twelve-well plates, each well containing glass coverslips, were coated with fibrinogen $(100 \mu \mathrm{g} / \mathrm{mL}$ overnight, $4^{\circ} \mathrm{C}$ ) and blocked (0.1\% BSA, 60 minutes, RT), and approximately $50 \times 10^{9} / \mathrm{L}$ platelets were placed into each well before LPC $(25 \mu \mathrm{M}), \mathrm{PC}(25 \mu \mathrm{M})$, PBS (negative control) or $\mathrm{ADP}$ (positive control, $20 \mu \mathrm{M}$ ) were added $\left(20\right.$ minutes, $37^{\circ} \mathrm{C}$ ). For the immunostaining, platelets were labelled with antiCD41 antibody and phalloidin for 30 minutes. After several washing steps, platelet attachment and spreading of 10 platelets per treatment were visualized microscopically by measuring the surfaces of the attached platelets (Olympus FSX100, Germany). 


\section{Platelet Aggregometry}

Washed platelets re-substituted with fibrinogen $(150 \mu \mathrm{g} / \mathrm{mL})$ were incubated with LPC or PC micelles, and light-transmission aggregometry (AggRAM, Helena Laboratories, United States) performed. Platelet samples were also pre-incubated with a P2Y 12 blocker (2MeSamp, $100 \mu \mathrm{M}$ ) before LPCs were added. Platelet aggregation was measured over 600 seconds and the area under the curve used for statistical analysis.

\section{Platelet Migration Assay}

Collagen-coated $\mu$-slide chemotaxis assays (ibidi, Germany) were used to investigate whether washed platelets migrate towards LPCs $(50 \mu \mathrm{M})$. Stromal cell-derived factor 1 (SDF-1) ( $3 \mu \mathrm{g} / \mathrm{mL}$, R\&D Systems, United States) was used as a positive control, and cell culture medium as a negative control. Platelet migration was video-recorded for up to 7 hours and migration direction and speed were analysed using ImageJ. Statistical analysis was performed with software from ibidi and the Rayleigh test was used to verify the level of significance of the directed movement.

\section{RNA Isolation and QRT-PCR}

RNA of DAMI cells and platelets was isolated using the TRIzol isolation method. Briefly, cells were incubated with trizol (5 minutes, RT) before chloroform was added. Cell lysates were centrifuged $\left(12,000 \times \mathrm{g}, 15\right.$ minutes, $\left.4^{\circ} \mathrm{C}\right)$ and the aqueous phase transferred into a new tube containing glycogen. RNA precipitation was induced by isopropanol (5 minutes, $4^{\circ} \mathrm{C}$ ) and precipitated RNA harvested by centrifugation $\left(12,000 \times g, 10\right.$ minutes, $\left.4^{\circ} \mathrm{C}\right)$. RNA-containing pellets were washed twice in $75 \% \mathrm{EtOH}$ and re-suspended in $10 \mu \mathrm{L}$ diethylpyrocarbonate-treated water. qRT-PCR was done with standard protocols and gene expression levels were calculated using the $\Delta \mathrm{CT}$ method with $18 \mathrm{~s}$ as the housekeeping gene. ${ }^{23}$

To investigate whether platelets express the LPC receptor G2AR, qRT-PCR of platelet complementary deoxyribonucleic acid was performed using commercial (Qiagen, Germany) as well as custom-made primers (Geneworks). qRT-PCR products were run on a $1.5 \%$ agarose gel and bands were visualized on a BIORAD (Gel Doc System, United States). As a negative control for G2AR expression HeLa cells, which are human fibroblastic cells, were used.

To investigate LPC-induced endothelial microRNA expression, human umbilical vein endothelial cells (HUVECs) were cultured under standard conditions and used at passage 4 before cells were stimulated with $50 \mu \mathrm{M} \mathrm{LPC}$ micelles or PBS for 6 hours. RNA isolation, qRT-PCR kits and primers were from Qiagen (Germany) and standard protocols were used.

\section{Mouse Model of Atherosclerotic Plaque Instability}

To assess LPC concentrations in stable and unstable atherosclerotic plaques, a recently developed tandem stenosis (TS) mouse model was used that reflects the characteristics of unstable, vulnerable atherosclerotic plaques as seen in humans. ${ }^{24,25}$ Briefly, 6-week-old apolipoprotein E-deficient $\left(\mathrm{ApoE}^{-1-}\right)$ mice were placed on a high-fat diet for 6 weeks before a TS with a $150-\mu \mathrm{m}$ outer diameter was introduced at the right carotid artery. The left carotid artery remained healthy and did not develop atherosclerosis. The aortic arch developed stable atherosclerosis. Mice were euthanized 8 weeks after surgery and vessel segments (right carotid artery and aortic arch), healthy arterial tissue (left carotid artery) and blood were collected for lipid analysis. For blood lipid analysis, a control group of $\mathrm{ApoE}^{-1-}$ mice were subjected to a sham operation (TS with $450 \mu \mathrm{m}$ TSs of the carotid artery) and their values compared with the lipid levels of mice with $150 \mu \mathrm{m}$ diameter stenoses in the TS model.

\section{Immunohistochemistry and Histochemical Staining of Mouse Tissue Slides}

Cryosections were obtained $500 \mu \mathrm{m}$ proximal to the proximal suture in the right common carotid artery of the TS mouse model representing unstable atherosclerotic plaques, the corresponding location in the left common carotid artery representing healthy arteries and from the aortic arch representing stable plaques. Haematoxylin and eosin (H\&E) staining was used for morphology. For Oil red O staining, frozen sections were fixed in $10 \%$ formalin, washed, stained in Oil red 0 solution ( $0.3 \%$ in isopropanol, Sigma) for 1 hour, differentiated in $60 \%$ isopropanol, dehydrated and mounted by Aquatex (Merck, United States). For CD68 staining, frozen section were incubated with primary antibody (Bio-Rad, United States) $1: 200$ at $4^{\circ} \mathrm{C}$ overnight. Following wash steps and incubation in avidin-biotin complex reagent, 3,3'-diaminobenzidine reagent was applied to each section. As soon as sections turned brown, slides were immersed in $\mathrm{dH}_{2} \mathrm{O}$ to stop the reaction. Slides were dehydrated and mounted on coverslips with dibutylphthalate polystyrene xylene mounting media. Images were obtained using a light microscope (Leica Brightfield microscope).

\section{Matrix-Assisted Laser Desorption Ionization and Histology}

After increased levels of different LPC species were found in mouse plaques, LPC distributions were investigated in human plaque using matrix-assisted laser desorption ionization (MALDI). Human atherosclerotic plaque samples were obtained from patients with symptomatic carotid artery stenosis who underwent carotid endarterectomy. All patients gave written informed consent and the study was approved by the ethics committee of the Alfred Hospital, Melbourne, Australia.

Plaque tissue samples were cryosectioned and fixed on slides before histology staining or MALDI imaging. To detect plaque areas that met the criteria of vulnerable, unstable atherosclerotic plaques, tissue slides were stained for markers known to be increased in unstable plaques. In particular, staining for matrix metallopeptidase 9 (MMP-9) (R\&D Systems) and collagen (Masson's trichrome staining) was performed using standard staining protocols. Corresponding sections of slides in which plaque instability was shown, were used for LPC detection with MALDI. Note that $30 \mathrm{~mL}$ of matrix solution $(25 \mathrm{mg} / \mathrm{mL}$ in 700:300:1 v/v/v ethanol, Merck, Kilsyth, VA, Australia; Milli-Q water: trifluoroacetic acid, Sigma-Aldrich, St Louis, Missouri, United States) was used 
for each slide. Microscope slides were mounted on a cardboard support $(35 \mathrm{~cm} \times 20 \mathrm{~cm}$ ) using sticky tape and placed against the back wall of a fume cupboard. Matrix solution was sprayed from a distance of $50 \mathrm{~cm}$ using an Eclipse HP-CS airbrush with a 0.3-mm nozzle (Anest Iwata, Yokohama, Japan) equipped with a Smart Jet Pro air compressor (Anest Iwata) operating at 0.3 $\mathrm{mPa}$. The matrix was applied in coats by passing the spray over the sample in a sweeping motion from left to right, then right to left, then back again over 3 seconds. Each coat was allowed to dry before another coat was applied. This process was repeated until $30 \mathrm{~mL}$ of the matrix solution had been sprayed onto the target.

All mass spectrometric analyses were performed on a SYNAPT HDMS (Manchester, United Kingdom) orthogonal geometry time-of-flight (TOF) mass spectrometer fitted with an intermediate pressure MALDI source. This instrument was equipped with an imaging mass spectrometry device located between the quadrupole and TOF mass analyser. Prior to all analyses, the mass spectrometer was mass calibrated over an $\mathrm{m} / \mathrm{z}$ range from 100 to 1,000 using a mixture of two poly(ethylene glycol) (PEG) standards (Sigma-Aldrich) with average molecular weights of 600 and $1,000 \mathrm{Da}$. PEG standards were prepared at $1 \mathrm{mg} / \mathrm{mL}$ in a $1: 1 \mathrm{v} / \mathrm{v}$ mixture of acetonitrile (Honeywell, Morristown, New Jersey, United States) and water, combined with $\alpha$ cyano-4-hydroxycinnamic acid (CHCA) matrix solution (CHCA at $3 \mathrm{mg} / \mathrm{mL}$ in $1: 1 \mathrm{v} / \mathrm{v}$ acetonitrile-water) and aqueous $\mathrm{NaCl}$ at $1 \mathrm{mg} / \mathrm{mL}$ (Sigma-Aldrich) at 5:5:1 v/v/v, then spotted onto a stainless steel MALDI target plate in $1 \mu \mathrm{L}$ aliquots and allowed to dry. PEG standards were mass analysed on the mass spectrometer in the positive ion $\mathrm{V}$-mode of operation and resultant data were used to mass calibrate.

\section{Statistics}

Comparison of two variables was done with the Student's $t$-test. Multiple comparisons were done with a one- or twoway analysis of variance and Tukey's or Sidak's, respectively, post hoc analysis (version 8, GraphPad Software, United States). If not stated otherwise, data are shown as the mean \pm standard error of the mean. All tests were two-tailed and $p$-values of $\leq 0.05$ are described as significant.

\section{Results}

\section{Platelet-Derived MV Characterization and Lipid Distribution in Comparison to Platelets and MVs of Different Origins}

PMVs were characterized using flow cytometry. After gating using forward and sideward light scatter, PMVs were characterized by binding of annexin $\mathrm{V}$, measuring the surface expression of phosphatidylserine, and the expression of CD41, CD61 and CD42b, the latter representing glycoprotein (GP) Ib known to be partially shed with platelet activation, which explains the lower expression level of GPIb compared with the other surface markers ( - Supplementary Fig. S1AS1I, available in the online version). ${ }^{26}$ In our lipidomic profiling, mass spectrometry revealed that MVs released from ADPstimulated platelets contained up to $3.4 \%$ LPC ( - Fig. 1A), while the LPC content of ADP-activated platelets was only 2.1\%, indicating an active packaging mechanism of LPC into platelet MVs (-Fig. 1B). MVs from THP-1 cells contained less LPC, $2.2 \%$ ( - Fig. 1C), as did MVs of the megakaryocytic cell line DAMI with 1.5\% LPC (-Fig. 1D). We also investigated whether the type of agonist used to activate platelets influences the LPC content of PMVs. In general, the LPC content in PMVs was higher than in activated platelets. Thrombin as the strongest platelet agonist increased the LPC content of platelet MVs more

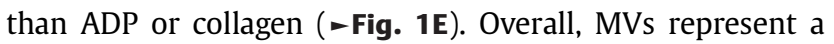
potential source of LPC in the circulation and potentially as deposits in the tissue.

\section{LPC Induces Inflammatory Changes in Endothelial Cells} It has been recently described that LPC induces intercellular adhesion molecule- 1 expression on human endothelial cells. ${ }^{27}$ As an additional measure of pro-inflammatory changes, we investigated potential changes induced by LPC focusing on a spectrum of microRNAs known to be regulated during endothelial dysfunction. ${ }^{28-30}$ MicroRNAs 19a, 34a, 132, 155 and 200c were all strongly increased after incubation of HUVECS with LPC (- Supplementary Fig. S2, available in the online version).

\section{LPC Activates Platelets and Induces Platelet- Monocyte Aggregates}

It is known that PMVs are involved in inflammation and coagulation. ${ }^{31}$ To investigate whether this is mediated by the high LPC content of PMVs, platelets were incubated with LPC or PC micelles, then platelet activation, as well as PMA formation, were assessed by flow cytometry. LPC induced significant activation of the platelet integrin GPIIb/IIla in comparison to PC $(10 \mu \mathrm{M}$ LPC vs. $10 \mu \mathrm{M}$ PC: $8.5 \pm 1.765$ vs. $16.33 \pm 1.085$, $p=0.025 ; 25 \mu \mathrm{M}$ LPC vs. $25 \mu \mathrm{M}$ PC: $114.7 \pm 28.29$ vs. $15.17 \pm 1.138, n=6, p=0.0173$, -Fig. 2A). Additionally, the platelet-activation marker CD62P was up-regulated in LPC-treated platelets $(10 \mu \mathrm{M}$ LPC vs. $10 \mu \mathrm{M}$ PC: $245.5 \pm 48.92$ vs. $76.17 \pm 21.22, p=0.048 ; 25 \mu \mathrm{M}$ LPC vs. $25 \mu \mathrm{M}$ PC: $1,522 \pm 388$ vs. $79.00 \pm 17.77, n=6, p=0.0147$ ) ( - Fig. 2B). Flow cytometry data showing LPC-induced platelet activation were confirmed in a proof-of-concept experiment demonstrating that LPC induces translocation of intracellularly stored CD62P to an extent comparable to ADP ( - Figs. 2C-F). CD62P (P-selectin) is the ligand of the monocyte receptor Pselectin glycoprotein ligand (PSGL)-1 and essential for the formation of highly inflammatory PMAs. In agreement, LPC significantly induced PMA formation (CD41/CD14 positive events in percentage of all CD14-positive events: PBS vs. LPC: $11 \pm 1.628 \%$ vs. $23.66 \pm 2.138 \%, p=0.022$; PBS vs. $\mathrm{PC}:$ $11 \pm 1.628 \%$ vs. $13.44 \pm 1.847 \%, p=$ n.s., $n=5$ ).

\section{LPC Induces Platelet Adhesion and Spreading}

To investigate whether LPC affects platelet adhesion, platelets were allowed to adhere to a fibrinogen matrix under static conditions. As shown in - Fig. 3A, LPC-activated platelets spread on fibrinogen-coated slides to an extent similar to that of ADP-stimulated platelets (-Fig. 3B). Significantly less platelet spreading was observed for PC- 


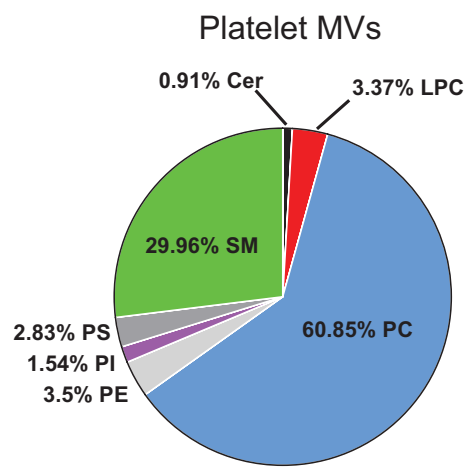

A

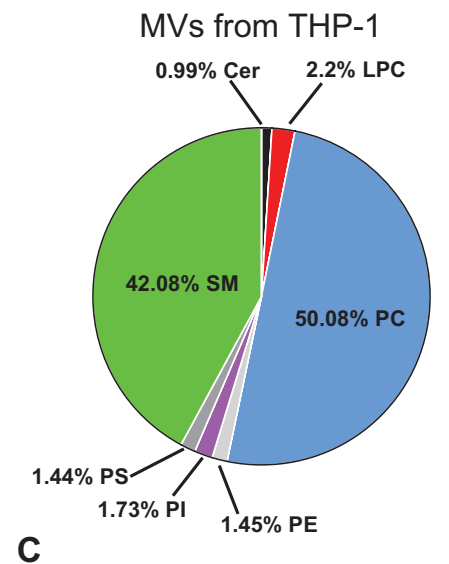

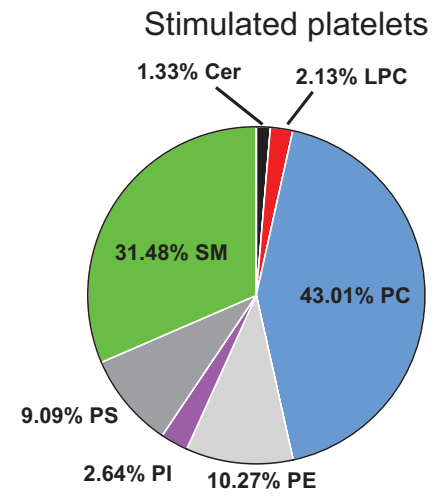

B

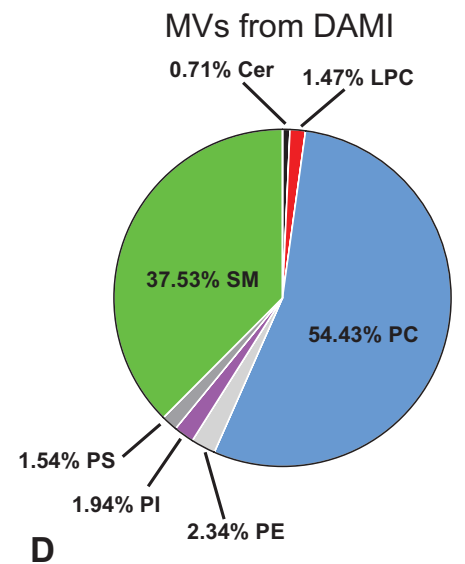

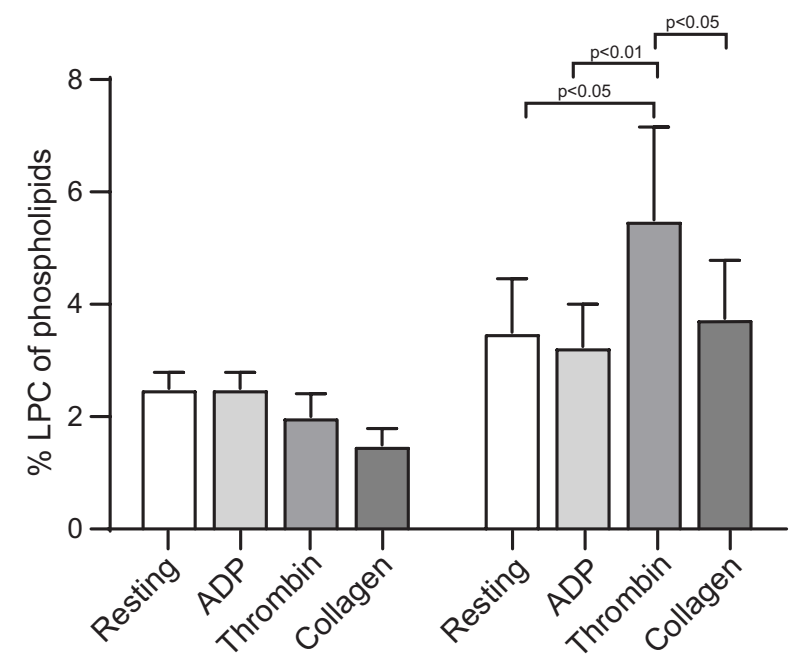

E

Platelets

\section{Platelet MVs}

Fig. 1 Lipid distribution in activated platelets and microvesicles (MVs) of different origins. (A) Platelet-derived MVs (PMVs) contain more LPC than activated platelets (B). In comparison to PMVs, MVs from THP-1 cells and DAMI cells contain less LPC (C and D). The mean of $n=3$ samples are given. The platelet agonists adenosine diphosphate (ADP), thrombin and collagen used to activate platelets and to generate PMVs all showed an overall statistically significant increased LPC content in PMVs compared with platelets. However, the individual agonist used did not result in a statistically significant difference in LPC content of PMVs (E). The overall comparison of platelet versus platelet MVs LPC content revealed that platelet MVs contain more LPC than the platelets from which they were released. $n=4$. Cer, ceramides; LPC, lysophosphatidylcholines; PC, phosphatidylcholines; PE, phosphatidylethanolamines; PI, phosphatidylinositols; PS, phosphatidylserines; SM, sphingomyelins.

treated ( - Fig. 3C) and PBS-incubated ( - Fig. 3D) platelets. In agreement, surface areas of LPC-incubated platelets were significantly larger than those of PC-incubated platelets (PBS vs. ADP vs. LPC vs. PC: $1,315 \pm 114$ vs. $3,509 \pm 333$ vs. $2,829 \pm 453$ vs. $866 \pm 73, n=10, p<0.0001$, - Fig. 3E).
Platelets attached to fibrinogen and those pre-treated with LPC or ADP showed strong formation of lamellipodia and filopodia in comparison to those incubated with PBS or PC (-Fig. 3F) as shown by anti-CD41 and phalloidin staining on platelets. 

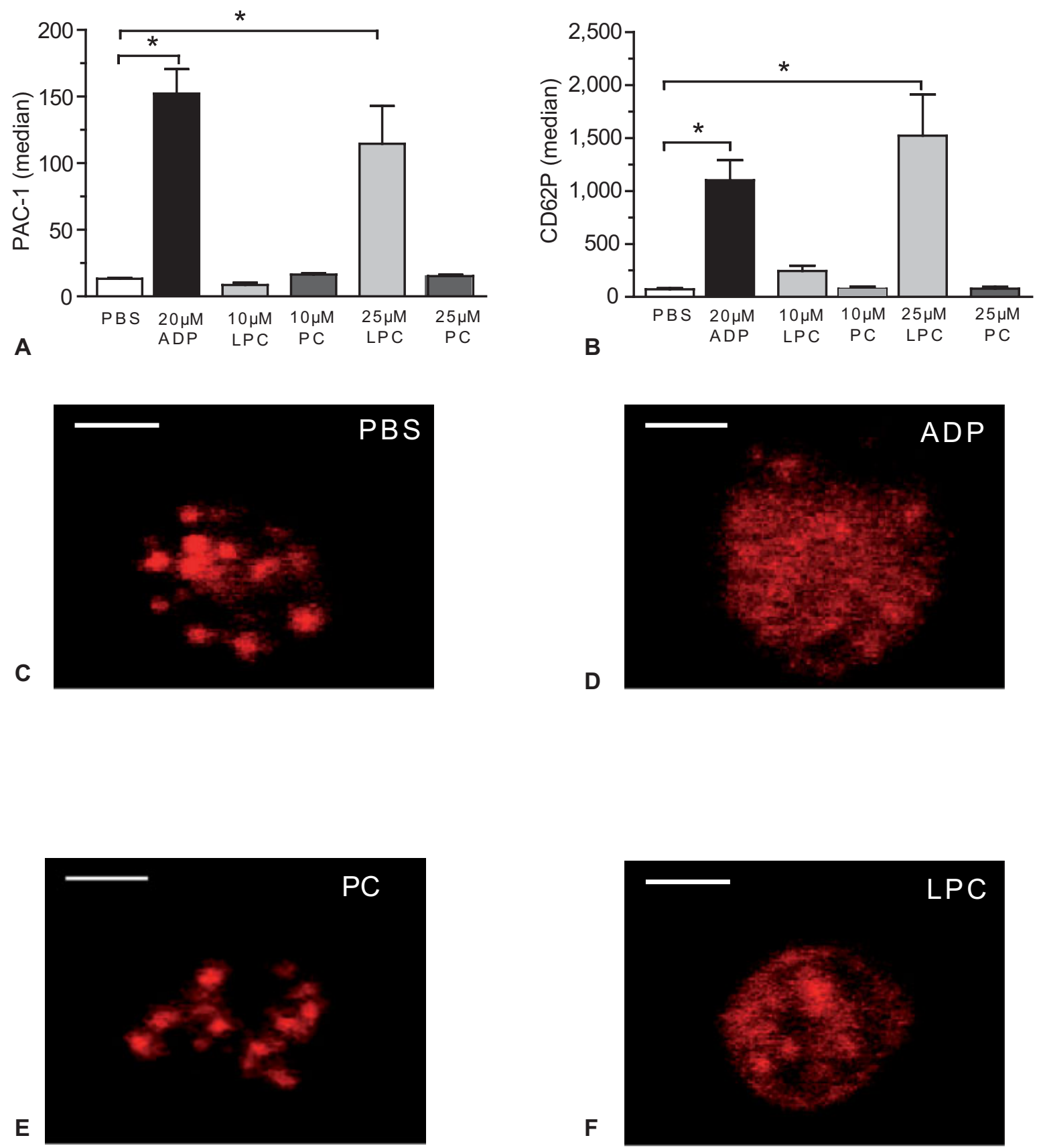

Fig. 2 Lysophosphatidylcholine (LPC) is a potent platelet activator. LPC but not phosphatidylcholine (PC) significantly induces activation of the platelet integrin glycoprotein (GP) Il $/ \mathrm{b} / \mathrm{ll}$ a, as detected by procaspase-activating compound 1 (PAC-1) binding in flow cytometry (A). Additionally, the platelet-activation marker CD62P is up-regulated after LPC treatment in flow cytometry (B). The effect of LPC on CD62P was confirmed in fluorescence microscopy with anti-CD62P staining, revealing that adenosine diphosphate (ADP) (D) and LPC (F), but not phosphate-buffered saline (PBS) (C) or PC (E), induce degranulation and translocation of CD62P. Bar indicates $2 \mu \mathrm{m} .{ }^{*} \mathrm{p}<0.05, n=3$.

\section{LPC Induces Platelet Aggregation}

Platelet aggregation is a critical step in atherothrombosis. To assess whether LPC affects platelet aggregation, light-transmission aggregometry was performed. LPC induced platelet aggregation to an extent similar to that of $20 \mu \mathrm{M}$ ADP (PBS vs. $50 \mu \mathrm{M}$ LPC: $3.752 \pm 1.874$ vs. $61.39 \pm 12.57, p=0.0011$; PBS vs. $50 \mu \mathrm{M}$ PC: $3.752 \pm 1.874$ vs. $3.193 \pm 1.869, p=0.6007$, $n=6$, - Figs. 4A and B). LPC-induced aggregation was also investigated in platelets pre-treated with a $\mathrm{P}_{2} \mathrm{Y}_{12}$ receptor blocker (2MeSamp). 2MeSamp did not affect LPC-induced platelet aggregation ( $50 \mu \mathrm{M}$ LPC $+2 \mathrm{MeSAMP}$ vs. $50 \mu \mathrm{M}$ LPC: $63.33 \pm 9.748$ vs. $61.39 \pm 12.57, n=6, p=0.7561$ ), indicating that LPC acts independently of the $\mathrm{P}_{2} \mathrm{Y}_{12}$ receptor.

\section{LPC Acts as a Chemoattractant for Platelet Migration}

Platelet migration is a more recently recognized component of platelet function. We adapted a migration assay and assessed the hypothesis that LPC acts as a chemoattractant. - Figs. 4C-E shows three migration assays demonstrating platelet migration towards SDF-1, which is a well-known platelet chemoattractant. 

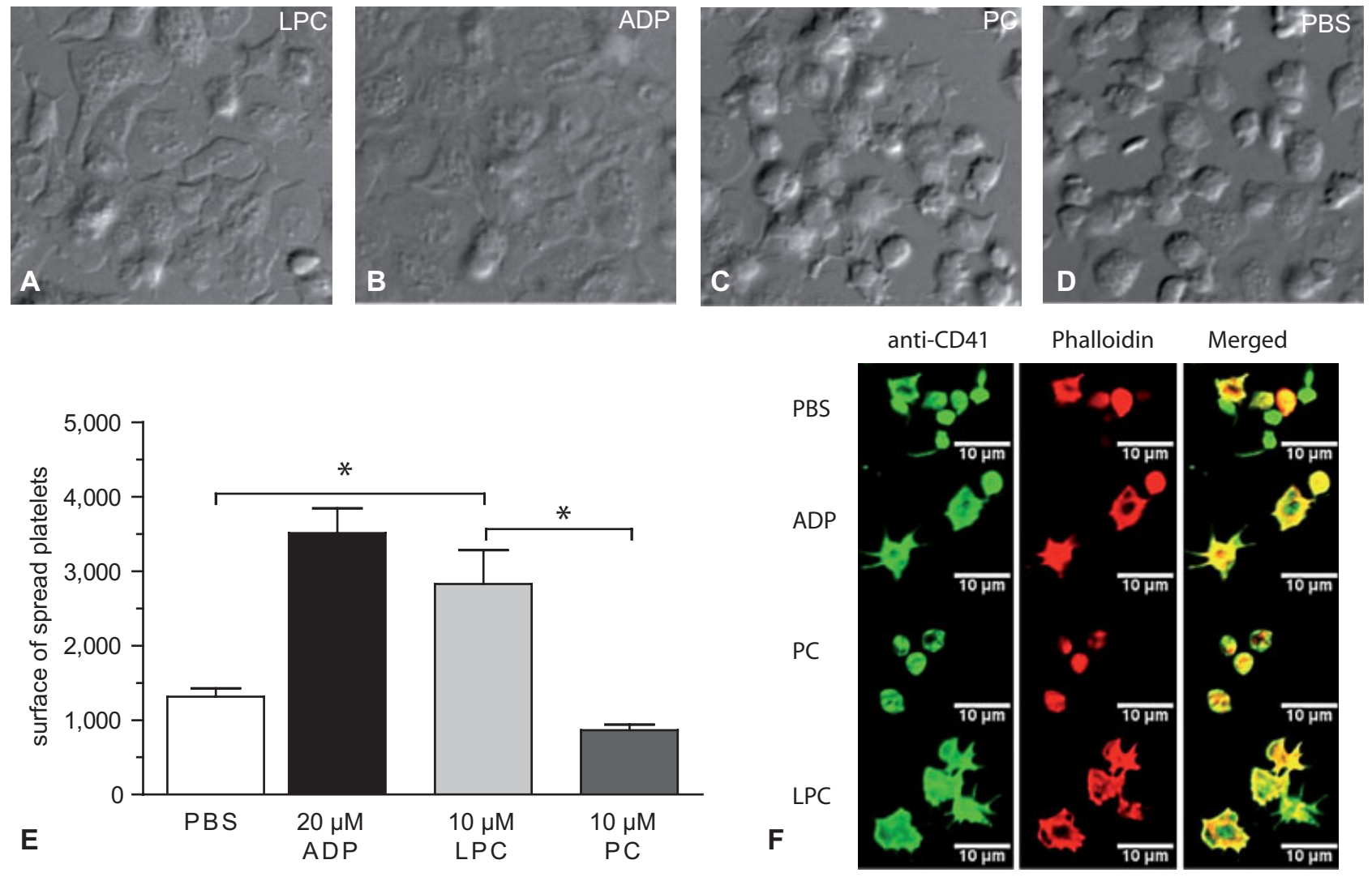

Fig. 3 Lysophosphatidylcholine (LPC) induces platelet adhesion and spreading to immobilized fibrinogen. (A) LPC-treated platelets show strong platelet adhesion and spreading on fibrinogen, similar to adenosine diphosphate (ADP) (B). In comparison to LPC and ADP, phosphatidylcholine (PC) (C) and phosphate-buffered saline (PBS) (D) cause significantly less platelet spreading. In agreement, LPC-treated platelets spread over a significantly larger surface area than platelets treated with $\mathrm{PC}\left(\mathrm{E},{ }^{*} p<0.05, n=10\right)$. Furthermore, platelets were treated with PBS (negative control), ADP (positive control), PC or LPC and platelet adhesion to fibrinogen as well as formation of platelet lamellipodia and filopodia was investigated. Stainings were performed with anti-CD41 and phalloidin. It was found that platelets after ADP and LPC treatment showed strong formation of platelet lamellipodia and filopodia, which was not found in platelets treated with PBS and PC (exemplary pictures out of 3 experiments are shown) (F).

Platelets stimulated with SDF-1 migrated with an average velocity of $178 \mu \mathrm{m} / \mathrm{h}$ and a mean distance of $198 \mu \mathrm{m}$. Platelets moved with an average velocity of $144 \mu \mathrm{m} / \mathrm{h}$ and a mean distance of $128 \mu \mathrm{m}$ from the intersection of the $x$-axis and $y$ axis towards high LPC concentrations (-Figs. 4I-K). The Rayleigh test showed a significant $(p<0.05)$ migration preference along the $x$-axis for the side on which the LPC or SDF- 1 was located. Platelets not influenced by any chemoattractant moved with an average speed of $184.5 \mu \mathrm{m} / \mathrm{h}$ and a mean distance of 124 $\mu \mathrm{m}$ but in different directions with no side preferred. No directional movement was observed when both chambers were filled with medium only (-Figs. $\mathbf{4 F}-\mathbf{H}$ ). The Rayleigh test showed no significant preference $(p>0.05)$.

\section{G2A Receptor Expression on Platelets}

After finding that LPC specifically acts on platelets, we assessed whether platelets express the LPC receptor G2AR. Using qRT-PCR, we demonstrated that platelets and the megakaryocyte cell line DAMI, used as a cell contamination-free control, expressed G2AR ( Fig. 5A). Human fibroblasts (HeLa cells) did not have relevant G2AR expression levels and were used as a negative control ( - Fig. 5B). Additionally, we showed G2AR expression in histological assessments of platelet thrombi (-Figs. 5C-G). Supporting these data, a commercially available anti-G2AR antibody inhibited the effect of LPC on platelet activation (CD62P expression) in a dose-dependent manner ( $p<0.0001, n=6$, - Fig. 5H).

\section{LPC is a Marker of Unstable Atherosclerosis in Mice}

To investigate whether LPC is associated with atherosclerosis, plasma from ApoE $\mathrm{E}^{-1-}$ mice with TS surgery, a mouse model of plaque instability, was measured in comparison to mice with sham surgery. Typical histological characteristics of unstable plaques were found with Oil Red O, H\&E and antiCD68 staining. As shown in - Fig. 6A, the unstable plaque demonstrates accumulation of macrophages in the plaque with a significant reduction of the vessel lumen.

We found the total LPC content in plasma was significantly increased in mice with TS representing plaque instability (mice with sham surgery vs. TS mice: $488 \pm 5 \mu \mathrm{M}$ vs. $970 \pm 27 \mu \mathrm{M}, p<0.0001, n=3$, -Fig. 6B). Furthermore, we investigated the LPC content in various vessel segments and found it was significantly increased in unstable plaque segments compared with stable plaque and healthy vessel segments (healthy arteries vs. stable plaques vs. unstable plaques: $0.111 \pm 0.014$ vs. $0.297 \pm 0.026$ vs. $0.392 \pm 0054$, $n=5, p=0.0039$, - Fig. $6 C$ ), indicating LPC represents a potential surrogate marker for unstable atherosclerosis. 


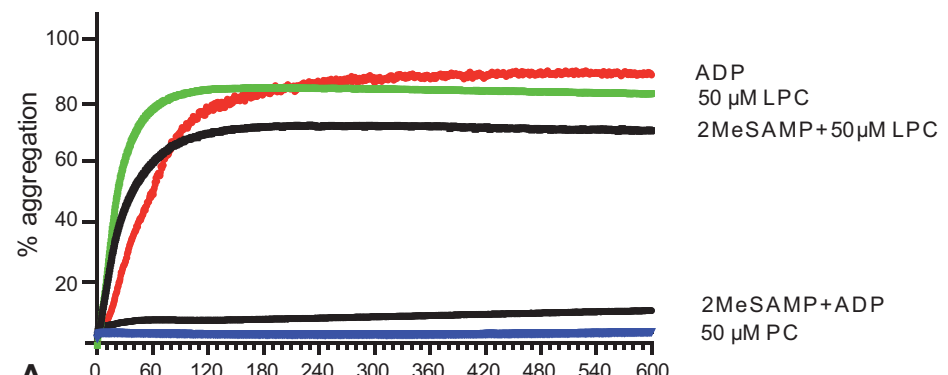

A

$\begin{array}{llllllllll}60 & 120 & 180 & 240 & 300 & 360 & 420 & 480 & 540 & 600\end{array}$ $50 \mu \mathrm{M} \mathrm{PC}$

time $[\mathrm{sec}]$

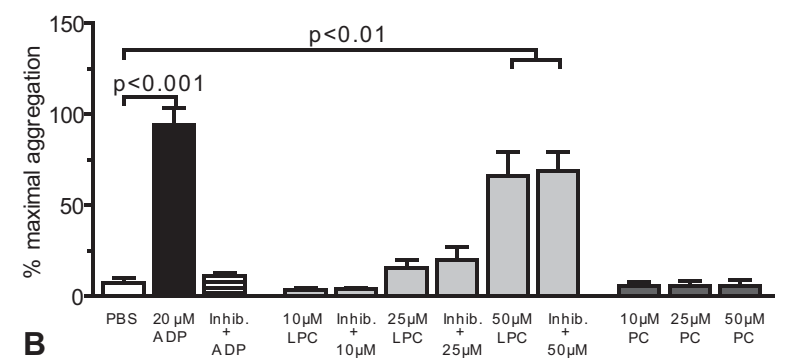

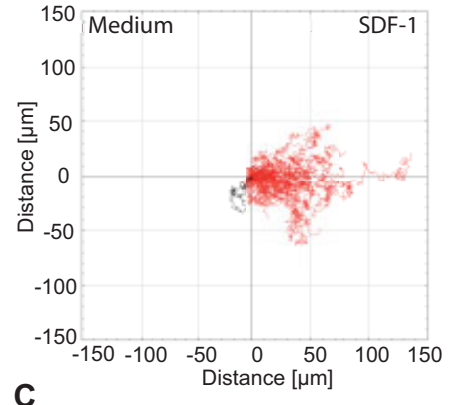

C

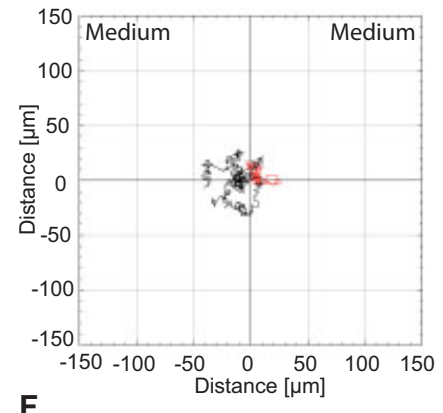

F

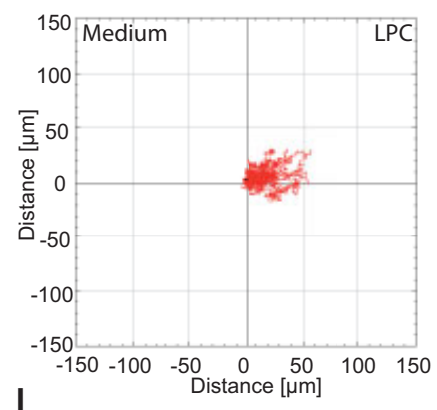

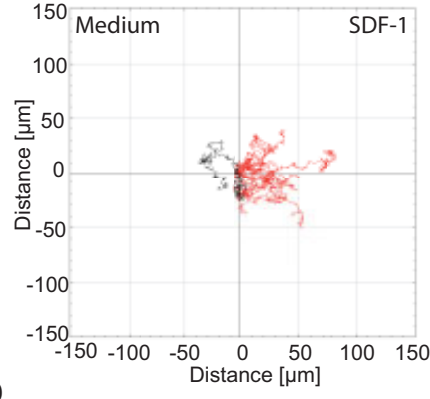

D

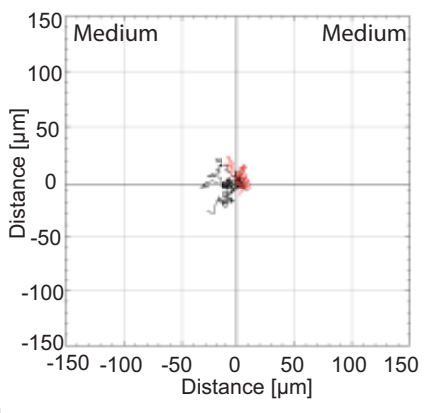

G

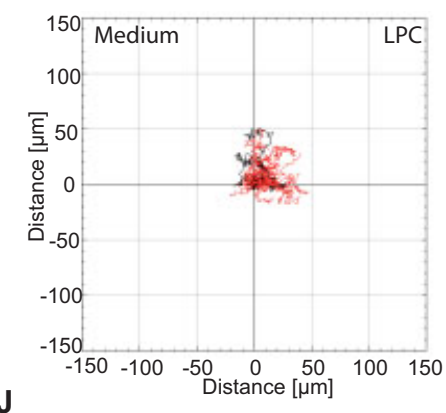

E
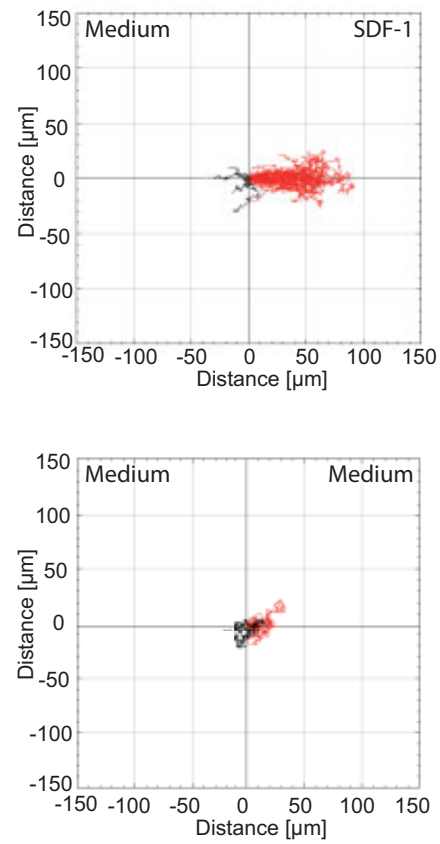

H

K

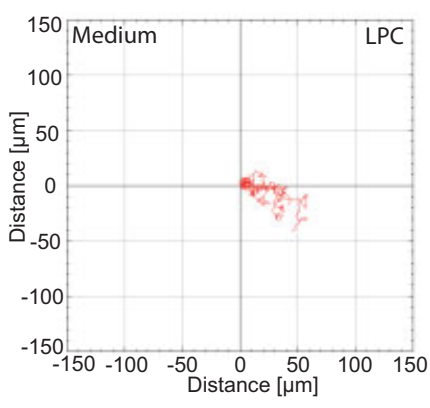

Fig. 4 Lysophosphatidylcholine (LPC) induces platelet aggregation and migration. As quantified by light-transmission aggregometry, LPC (green line) induces platelet aggregation to a similar extent as that of adenosine diphosphate (ADP) (red line), whereas phosphatidylcholine (PC) (blue line) has no effect on platelet aggregation (A). P2 $\mathrm{Y}_{12}$ inhibition by 2 MeSAMP (black line) does not affect LPC-induced platelet aggregation (B), indicating that LPC activates platelets independently of $\mathrm{P}_{2} \mathrm{Y}_{12}$. PC as a control does not induce platelet aggregation. ${ }^{*} p<0.05, n=6$. Using a $\mu$-slide migration assay, we found washed platelets migrate towards higher stromal cell-derived factor 1 (SDF-1) concentrations (positive control) (C-E). No directional migration of platelets towards medium without any chemoattractant (negative control) was observed (F-H). Platelets migrate directional towards LPC (I-K). 

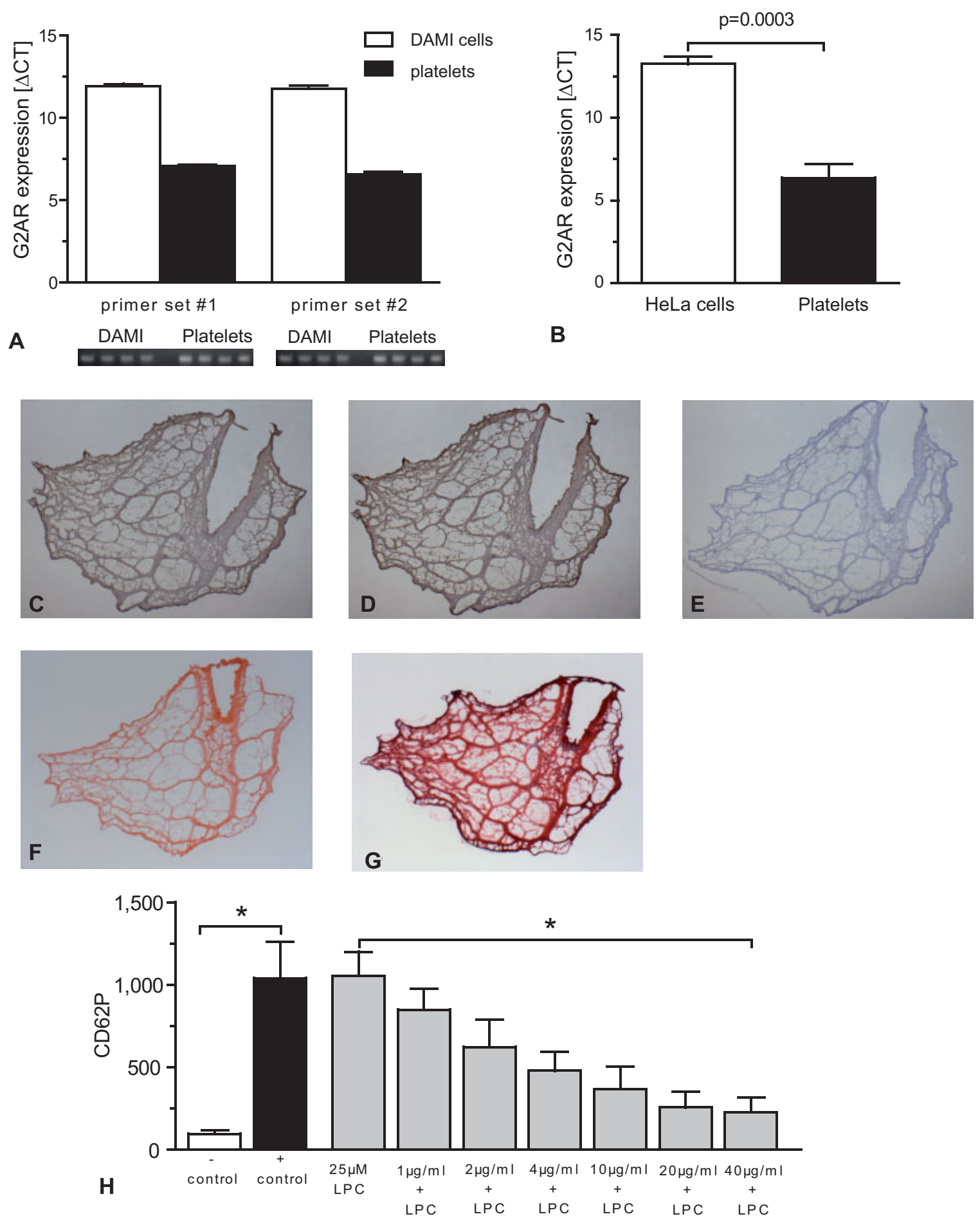

Fig. 5 Platelets express the lysophosphatidylcholine (LPC) receptor G2A. Using quantitative reverse transcription-polymerase chain reaction (qRT-PCR) with two different G2AR primer sets, we found that washed platelets, as well as the megakaryocytic cell line DAMI, express significant levels of G2AR (A, $n=4)$. Human HeLa cells were used as a negative control and did not show significant G2A receptor expression ( $n=4)$ (B). As shown via immunohistochemistry, thrombi express G2AR (C) and CD41 (D). (E) Phosphate-buffered saline (PBS) control (F) haematoxylin and eosin staining. (G) Masson's trichrome staining. Each of these stainings is representative of three independent stainings. Use of a specific G2ARblocking antibody significantly reduces LPC-induced CD62P expression on platelets in flow cytometry in a dose-dependent manner (doses from 1 to $40 \mu \mathrm{g} / \mathrm{mL}$ shown); indicating LPC acts on G2AR on platelets $(\mathrm{H}) .{ }^{*} p<0.05, n=6$.

\section{LPC Distributions in Human Unstable Plaques}

To confirm the data from the mice experiments and to indicate translational relevance, LPC content and local distribution were for the first time assessed in human plaques using MALDI.
To understand the localization of LPC in the human plaques, additional sections were stained with Masson's trichrome ( $\mathbf{- F i g}$. 7A) as well as immunohistochemistry staining with MMP-9 ( - Fig. 7B), demonstrating extracellular protease activities, which is also considered a marker of 

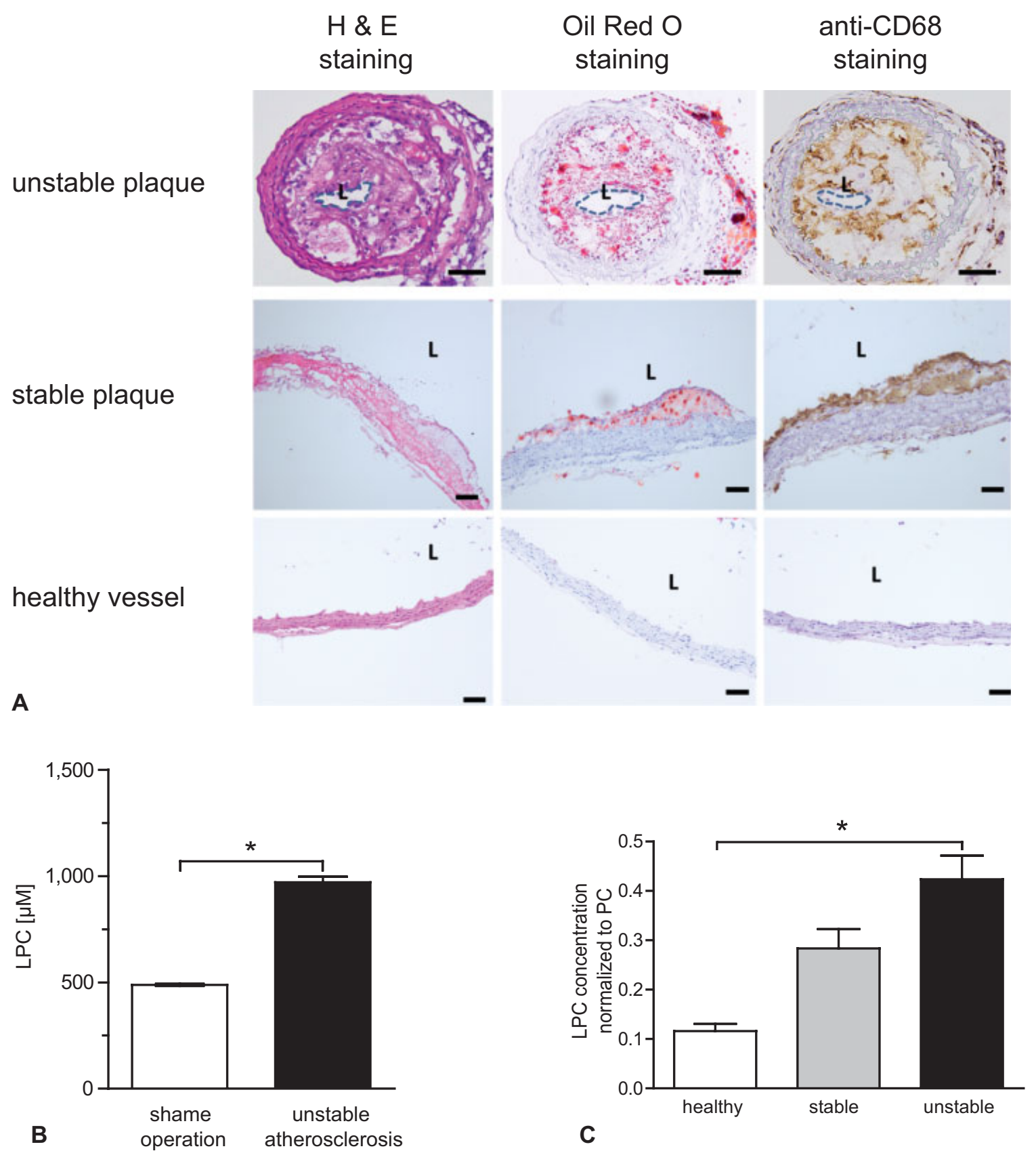

Fig. 6 Lysophosphatidylcholine (LPC) in blood of atherosclerotic mice and in unstable and stable atherosclerotic plaques of mice. Blood and tissue of healthy vessels, stable atherosclerotic plaques and unstable atherosclerotic plaques were characterized and investigated for their LPC contents. Oil Red O, haematoxylin and eosin (H\&E) and anti-CD68 staining of tissue slides from the tandem stenosis mouse model of plaque instability/rupture showed the typical accumulation of macrophages in the unstable plaque with a significant reduction of the vessel lumen (A). Blood of mice with unstable atherosclerosis contains significantly higher amounts of LPC than those with stable atherosclerosis (B). Furthermore, LPC concentrations in murine vessels with stable atherosclerotic lesions are higher than those of healthy arterial tissue, while they are the highest in arteries with unstable atherosclerotic plaques (C). ${ }^{*} p<0.05, n=3$ for blood analysis and $n=5$ for vessel tissue analysis. $L$ indicates the vessel lumen, bar indicates $100 \mu \mathrm{m}$.

plaque instability. MALDI imaging revealed that the most abundant LPC species, LPC 16:0 (-Fig. 7C), LPC 18:0 (-Fig. 7D) and LPC 18:1 (-Fig. 7E), were enriched in the necrotic cores of atherosclerotic plaques.

\section{Discussion}

The major findings of the study are: (1) LPC is a major lipid of PMVs. (2) LPC induces platelet activation, aggregation, adhe- sion, spreading and migration, as well as the formation of PMAs. (3) G2AR is expressed on platelets and mediates the effects of LPC on platelets. (4) LPC is increased in plasma of mice with unstable atherosclerosis. (5) LPC is highly abundant in unstable atherosclerotic plaques in both mice and humans.

Increasing evidence has emerged that MVs are important biovectors that transfer cellular components (e.g. proteins, cell organelles, RNA) to their target cells, thereby mediating 

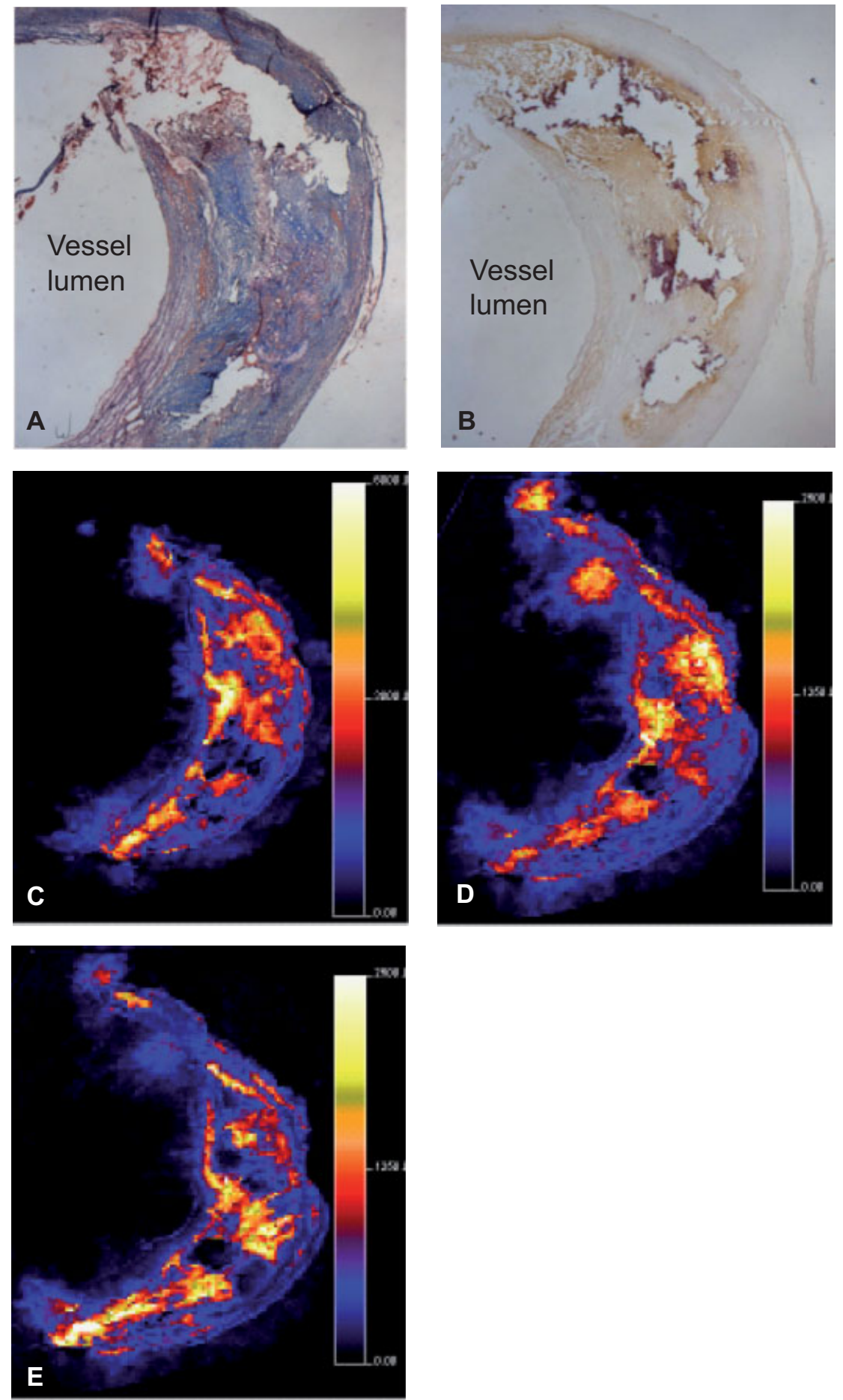

Fig. 7 Lysophosphatidylcholine (LPC) mapping of human vulnerable plaques. To investigate the morphology of atherosclerotic human plaque, tissue slides were stained for collagen (A) and matrix metallopeptidase 9 (MMP-9) (B). LPC 16:0 (C), 18:0 (D) and 18:1 (E) content was determined in these tissue sections. LPC mapping of human vulnerable plaques shows LPC concentrations are highest in areas of plaque vulnerability.

strong inflammation and coagulation in acute and chronic

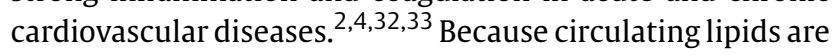
involved in atherogenesis, we assessed whether the inflammatory effect of MVs can be attributed to the transport of inflammatory lipids such as LPC. ${ }^{34}$ LPC is one of the main bioactive molecules in oxidized low-density lipoprotein and is involved in several conditions associated with vascular oxidative stress and inflammation. ${ }^{35,36}$ However, as LPC is promptly metabolized in circulation by serum lysophospholipases, LPC is typically found to be associated with carrier proteins. ${ }^{37}$ In this study, we demonstrate that PMVs, the most abundant type of MVs circulating in blood, contain large amounts of LPC and represent an important vehicle for LPC. Interestingly, these MVs contain relatively more LPC than do activated platelets, suggesting an active packaging mechanism of LPC into MVs. 
PMVs are postulated to have strong pro-coagulant effects. ${ }^{38}$ Hypothesizing these effects might be related to their LPC content, we found LPC causes activation of the platelet integrin GPIIb/IIIa, as well as increased surface expression of CD62P (P-selectin). Additionally, as shown by confocal microscopy, LPC induces the translocation of CD62P within the platelet to an extent similar to ADP. Several studies have proven that platelet CD62P interacts with the monocyte surface receptor PSGL-1, facilitating the formation of highly inflammatory PMAs. ${ }^{39,40}$ In agreement, we found LPC additionally increases PMA formation. These data indicate that LPC is a central component of PMVinduced platelet activation and platelet-monocyte crosstalk in cardiovascular disease.

Atherothrombotic complications are feared in patients with coronary artery disease and platelet activation plays a major role in these events as well as throughout atherogenesis. ${ }^{41,42}$ We hypothesized that LPC is a mediator of platelet activation in atherothrombotic events. Interestingly, it was reported that Trypanosoma cruzi, the cause of life-threatening Chagas disease, induces platelet aggregation in an LPCdependent manner. ${ }^{43}$ We found that LPC induces platelet activation, aggregation and spreading, confirming its central role. We then aimed to identify the platelet receptor mediating these LPC effects on platelets. It is well known that LPC binds to the $G$ protein-coupled membrane receptor G2AR, which is expressed on a wide range of immune cells (e.g. lymphocytes and macrophages). This interaction mediates neutrophil and macrophage activation, as well as macrophage migration, and so is essential for both innate and adaptive immunity. However, to the best of our knowledge, the expression of G2AR in platelets has never before been reported. Using PCR, we found significant levels of G2AR in platelets, as well as the megakaryocytic cell line DAMI, suggesting the LPC effect on platelets is mediated by G2AR. Supporting this, histological staining of platelet thrombi confirmed significant G2AR expression and G2AR inhibition significantly reduced platelet activation by LPC in a dosedependent manner.

It is evident that circulating PMVs bind to leukocytes and activate them. ${ }^{44}$ Gómez-Muñoz et al found that LPC induced phospholipase D in mouse macrophages, which promoted the synthesis of inflammatory second messengers such as phosphatidic acid, lysophosphatidic acid and diacylglycerol, and thereby contributed to the cellular response involved in atherosclerosis and inflammation. ${ }^{45}$ These data were confirmed by Huang et al, who described how LPC enhanced interferon $\gamma$ secretion of peripheral blood monocytes, as well as antibody production. ${ }^{46}$ Thus, once bound to circulating leukocytes or macrophages, platelet MVs might deliver their LPC to these cells, contributing to their activation and ultimately to vascular inflammation.

MVs of different cellular origin such as PMVs and T cellderived MVs have been found to be involved in atherogenesis. However, their impact on the progression of atherosclerotic lesions still remains controversial. ${ }^{47}$ Additionally, recent data indicate that also endothelial cells and smooth muscle cells of atherosclerotic plaques can release MV. ${ }^{48}$
Whether atherogenesis and plaque instability is mainly driven by circulating MVs attaching to the plaque or by MVs released from the plaque itself also needs to be elucidated in future studies.

It has been suggested that oxidized lipids as components of MVs are involved in monocyte-endothelial cell interactions and thus in vascular inflammation at the early stages of atherosclerosis. ${ }^{49}$ Otherwise, the impact of PMVs and their lipids on the progression of atherosclerosis is not well understood. In this study, enhanced levels of LPC were found in the plasma of mice exposed to unstable atherosclerotic plaques. These data imply that LPC in circulating MVs can be used as a marker for plaque instability.

A plasma risk marker of atherosclerotic plaque instability is highly sought-after and several advanced 'omics' approaches have been applied towards the discovery of such a marker. ${ }^{50}$ Lipidomics as applied in our characterization of MVs and platelets has only become available based on the technical advances in liquid chromatography and mass spectrometry systems over the recent years. Recently, Chatterjee et al reported lipidomics of platelets from patients with coronary artery disease and acute coronary syndromes allowing the identification of specific patterns of lipids associated with cardiovascular risk. ${ }^{51}$ Our work extends this approach using lipidomics on MVs, which has not been described before. Increasingly high-throughput capabilities, increased sensitivities and the extension of detectable lipid species make lipidomics of platelets and/ or MVs an attractive option for cardiovascular biomarker discovery.

Hypothesizing that PMVs are a potential source of LPC in circulation, our finding is in line with reports that PMVs are more abundant in atherosclerosis than controls. ${ }^{52}$ Our data are supported by those of Lavi et al, who found enhanced levels of LP-PLA2, the enzyme that metabolizes PC to LPC, and increased amounts of LPC, in patients with coronary artery disease. ${ }^{53}$ Wilensky et al found therapeutic inhibition of Lp-PLA2 with darapladib significantly reduced coronary atherosclerosis. ${ }^{54}$

PMVs are not only markers of atherosclerosis in the circulation but were also found to accumulate in atherosclerotic lesions. ${ }^{52,55}$ Using fluorescent angioscopy, LPC has been detected ex vivo in coronary artery plaques. ${ }^{56}$ These data were supported by Ménégaut et al, who reported ex vivo increased levels of 2-arachidonoyl-lysophosphatidylcholine in carotid atheroma plaques of diabetic patients. ${ }^{57}$ In agreement with these report, we found LPC to be more abundant in atherosclerotic plaques compared with normal arterial tissues.

Our unique LPC mapping of vulnerable plaques using MALDI imaging is consistent with a role of LPC in plaque instability. Our finding is also consistent with a previous lipidomic comparison of unstable and stable plaque tissue describing an accumulation of LPC in unstable atherosclerotic plaques. ${ }^{58}$ Similarly, it was shown that plaques from patients with symptomatic carotid artery stenosis contained more LPC than those of asymptomatic patients. ${ }^{59,60}$ These findings are also in line with a study by Herrmann et al, who 
showed Lp-PLA2 was highly expressed in carotid artery plaques and furthermore predicted the risk for cardiovascular complications. ${ }^{61}$ Despite this ample evidence of LPC accumulation in unstable plaques, it has to be acknowledged that the source of LPC in plaques is not yet defined. MVs are shown to be abundant in plaques, ${ }^{33}$ as such they are a potential source of plaque-associated LPC. However, further lipid tracing studies are warranted to ultimately clarify the source of unstable plaque-associated LPC.

Using MALDI to map LPC distribution in human unstable carotid plaque sections, we further found LPC was particularly enriched in necrotic plaque areas, those areas that were highly inflamed and prone to rupture. Considering that LPC in atherosclerotic plaques attracts further monocytes, which contain high levels of phospholipase A2 and thus increase LPC levels inside the plaque, LPC might in fact be a key contributor to plaque instability, promoting a vicious cycle of monocyte recruitment with consequently increased PLA2 and LPC levels. ${ }^{62}$ These data are supported by a finding that LPC itself induces macrophage polarization towards inflammatory M1 macrophages. ${ }^{63}$

Peter et al showed that LPC is a strong chemoattractant for macrophages, leading to macrophage accumulation at atherosclerotic sites of high LPC content and thus promoting further plaque instability. ${ }^{64}$ Within the last decade, it has become evident that platelets also have the ability to migrate towards chemokines, opening a whole new view into platelet (patho)physiology. ${ }^{65}$ As a follow-up of our findings of increased LPC concentration in unstable atherosclerotic plaques, and identification of G2AR as an LPC receptor on platelets, we assessed whether LPC acts as a chemoattractant for platelets. We found that platelets migrate towards increasing LPC concentrations. These data suggest that platelets migrate into areas of unstable, vulnerable plaques and thereby promote plaque inflammation.

At present, the intracellular signalling cascade on which LPC acts can only be hypothesized. Stafford et al found the protein kinase D2, an enzyme essential for intracellular LPC signalling in leukocytes, is also expressed in platelets. Therefore, the intracellular pathway by which LPC act on platelets could be similar to that of leukocytes. ${ }^{66}$ Moreover, recent data indicate exogenous LPC increases intracellular $\mathrm{Ca}^{2+}$ levels by opening transmembrane $\mathrm{Ca}^{2+}$ channels and by mobilizing $\mathrm{Ca}^{2+}$ from intracellular stores. ${ }^{67}$ Both mechanisms are vital for platelet activation and aggregation. Schmidt et al showed platelets migrated towards increased SDF-1 concentration, and $\mathrm{Ca}^{2+}$ ion channels were essential for this platelet migration. ${ }^{68} \mathrm{As} \mathrm{LPC}$ is known to increase intracellular $\mathrm{Ca}^{2+}$ concentrations, this may in fact be the underlying mechanism to explain why LPC acts as a chemoattractant for platelets.

In conclusion, PMVs are a major source of LPC in circulation. Binding of LPC to G2AR on platelets results in platelet activation, adhesion, spreading, migration and aggregation, as well as in the formation of PMAs. Additionally, LPC in circulating MVs in mice represents a marker for atherosclerotic plaque instability. Two-dimensional MALDI imaging/mapping in human atherosclerotic plaques shows LPC is particularly enriched in areas of plaque instability, where it may contribute to plaque inflammation.

\section{What is known about this topic?}

- Platelet-derived microvesicles are involved in vascular inflammation.

- LPC is a pro-inflammatory lipid that promotes activation of white blood cells.

- Activated platelets contribute to atherothrombosis with consecutive thromboembolic complications.

\section{What does this paper add?}

- Lysophosphatidylcholine (LPC) is a major component of platelet-derived microvesicles.

- LPC binds to the G2A receptor, which was newly identified to be expressed on platelets, and thereby causes platelet activation, spreading, migration and aggregation.

- Circulating LPC is a surrogate marker for plaque instability in mice and was significantly increased in vulnerable areas of atherosclerotic plaques, in both mice and humans.

Note

An abstract of this paper was presented at the ISTH Congress, July 6-10, 2019 in Melbourne, Australia.

Funding

P.D. was funded by scholarships from the German Research Foundation (DFG, DI 1623/1-1) and Monash University in Melbourne (Australia), J.D.M. and N.M.H. were funded by Royal Australasian College of Physicians research establishment fellowships, S.U.E. was funded by a professorial Heisenberg Fellowship from the German Research Foundation (DFG) and X.W. and Y.C.C. were funded by a fellowship from the Heart Foundation of Australia. P.J.M. and K.P. were funded by fellowships from the National Health and Medical Research Council (NHMRC) of Australia. The project was also supported in part by the Victorian Government's OIS Program.

Conflict of Interest

None declared.

Acknowledgements

We thank Jacquie Weir, Alba Fricke and Laura Sander for technical assistance.

\section{References}

1 Yuana Y, Sturk A, Nieuwland R. Extracellular vesicles in physiological and pathological conditions. Blood Rev 2013;27(01):31-39 
2 Zaldivia MTK, McFadyen JD, Lim B, Wang X, Peter K. Plateletderived microvesicles in cardiovascular diseases. Front Cardiovasc Med 2017;4:74

3 Ridger VC, Boulanger CM, Angelillo-Scherrer A, et al; Position Paper of the European Society of Cardiology (ESC) Working Group on Atherosclerosis and Vascular Biology. Microvesicles in vascular homeostasis and diseases. Thromb Haemost 2017;117(07):1296-1316

4 Hugel B, Martínez MC, Kunzelmann C, Freyssinet JM. Membrane microparticles: two sides of the coin. Physiology (Bethesda) 2005; 20:22-27

5 Pluskota E, Woody NM, Szpak D, et al. Expression, activation, and function of integrin alphaMbeta2 (Mac-1) on neutrophil-derived microparticles. Blood 2008;112(06):2327-2335

6 Diehl P, Fricke A, Sander L, et al. Microparticles: major transport vehicles for distinct microRNAs in circulation. Cardiovasc Res 2012;93(04):633-644

7 Brown GT, McIntyre TM. Lipopolysaccharide signaling without a nucleus: kinase cascades stimulate platelet shedding of proinflammatory IL-1ß-rich microparticles. J Immunol 2011;186(09): 5489-5496

8 Mause SF, von Hundelshausen P, Zernecke A, Koenen RR, Weber C. Platelet microparticles: a transcellular delivery system for RANTES promoting monocyte recruitment on endothelium. Arterioscler Thromb Vasc Biol 2005;25(07):1512-1518

9 Bernal-Mizrachi L, Jy W, Jimenez JJ, et al. High levels of circulating endothelial microparticles in patients with acute coronary syndromes. Am Heart J 2003;145(06):962-970

10 Bei Y, Das S, Rodosthenous RS, et al. Extracellular vesicles in cardiovascular theranostics. Theranostics 2017;7(17):4168-4182

11 Vajen T, Benedikter BJ, Heinzmann ACA, et al. Platelet extracellular vesicles induce a pro-inflammatory smooth muscle cell phenotype. J Extracell Vesicles 2017;6(01):1322454

12 Edelstein LC. The role of platelet microvesicles in intercellular communication. Platelets 2017;28(03):222-227

13 Vajen T, Mause SF, Koenen RR. Microvesicles from platelets: novel drivers of vascular inflammation. Thromb Haemost 2015;114 (02):228-236

14 Burnier L, Fontana P, Kwak BR, Angelillo-Scherrer A. Cell-derived microparticles in haemostasis and vascular medicine. Thromb Haemost 2009;101(03):439-451

15 Mause SF, Weber C. Microparticles: protagonists of a novel communication network for intercellular information exchange. Circ Res 2010;107(09):1047-1057

16 Stegemann C, Pechlaner R, Willeit P, et al. Lipidomics profiling and risk of cardiovascular disease in the prospective population-based Bruneck study. Circulation 2014;129(18):1821-1831

17 Braig D, Nero TL, Koch HG, et al. Transitional changes in the CRP structure lead to the exposure of proinflammatory binding sites. Nat Commun 2017;8:14188

18 Habersberger J, Strang F, Scheichl A, et al. Circulating microparticles generate and transport monomeric C-reactive protein in patients with myocardial infarction. Cardiovasc Res 2012;96(01):64-72

19 Weir JM, Wong G, Barlow CK, et al. Plasma lipid profiling in a large population-based cohort. J Lipid Res 2013;54(10):2898-2908

20 Elizondo E, Moreno E, Cabrera I, et al. Liposomes and other vesicular systems: structural characteristics, methods of preparation, and use in nanomedicine. Prog Mol Biol Transl Sci 2011;104:1-52

21 Greenberg SM, Rosenthal DS, Greeley TA, Tantravahi R, Handin RI. Characterization of a new megakaryocytic cell line: the Dami cell. Blood 1988;72(06):1968-1977

22 Schwarz M, Meade G, Stoll P, et al. Conformation-specific blockade of the integrin GPIIb/IIIa: a novel antiplatelet strategy that selectively targets activated platelets. Circ Res 2006;99(01):25-33

23 Rikitake Y, Hirata K, Yamashita T, et al. Expression of G2A, a receptor for lysophosphatidylcholine, by macrophages in murine, rabbit, and human atherosclerotic plaques. Arterioscler Thromb Vasc Biol 2002;22(12):2049-2053
24 Chen YC, Bui AV, Diesch J, et al. A novel mouse model of atherosclerotic plaque instability for drug testing and mechanistic/ therapeutic discoveries using gene and microRNA expression profiling. Circ Res 2013;113(03):252-265

25 Htun NM, Chen YC, Lim B, et al. Near-infrared autofluorescence induced by intraplaque hemorrhage and heme degradation as marker for high-risk atherosclerotic plaques. Nat Commun 2017; $8(01): 75$

26 Montague SJ, Andrews RK, Gardiner EE. Mechanisms of receptor shedding in platelets. Blood 2018;132(24):2535-2545

27 Li X, Fang P, Li Y, et al. Mitochondrial reactive oxygen species mediate lysophosphatidylcholine-induced endothelial cell activation. Arterioscler Thromb Vasc Biol 2016;36(06):1090-1100

28 Qin B, Yang H, Xiao B. Role of microRNAs in endothelial inflammation and senescence. Mol Biol Rep 2012;39(04):4509-4518

29 Hackl M, Brunner S, Fortschegger K, et al. miR-17, miR-19b, miR20a, and miR-106a are down-regulated in human aging. Aging Cell 2010;9(02):291-296

30 Hergenreider E, Heydt S, Tréguer K, et al. Atheroprotective communication between endothelial cells and smooth muscle cells through miRNAs. Nat Cell Biol 2012;14(03):249-256

31 Puddu P, Puddu GM, Cravero E, Muscari S, Muscari A. The involvement of circulating microparticles in inflammation, coagulation and cardiovascular diseases. Can J Cardiol 2010;26(04): 140-145

32 Shantsila E, Kamphuisen PW, Lip GY. Circulating microparticles in cardiovascular disease: implications for atherogenesis and atherothrombosis. J Thromb Haemost 2010;8(11):2358-2368

33 Boulanger CM, Loyer X, Rautou PE, Amabile N. Extracellular vesicles in coronary artery disease. Nat Rev Cardiol 2017;14 (05):259-272

34 Yuan Y, Li P, Ye J. Lipid homeostasis and the formation of macrophage-derived foam cells in atherosclerosis. Protein Cell 2012;3(03):173-181

35 Ketelhuth DF, Hansson GK. Cellular immunity, low-density lipoprotein and atherosclerosis: break of tolerance in the artery wall. Thromb Haemost 2011;106(05):779-786

36 Martinet W, Schrijvers DM, De Meyer GR. Necrotic cell death in atherosclerosis. Basic Res Cardiol 2011;106(05):749-760

37 Ojala PJ, Hermansson M, Tolvanen M, et al. Identification of alpha1 acid glycoprotein as a lysophospholipid binding protein: a complementary role to albumin in the scavenging of lysophosphatidylcholine. Biochemistry 2006;45(47):14021-14031

38 Sinauridze EI, Kireev DA, Popenko NY, et al. Platelet microparticle membranes have 50 - to 100 -fold higher specific procoagulant activity than activated platelets. Thromb Haemost 2007;97(03): 425-434

39 McGregor L, Martin J, McGregor JL. Platelet-leukocyte aggregates and derived microparticles in inflammation, vascular remodelling and thrombosis. Front Biosci 2006;11:830-837

40 McEver RP, Cummings RD. Role of PSGL-1 binding to selectins in leukocyte recruitment. J Clin Invest 1997;100(11, Suppl): S97-S103

41 Kaplan ZS, Jackson SP. The role of platelets in atherothrombosis. Hematology (Am Soc Hematol Educ Program) 2011;2011:51-61

42 Massberg S, Brand K, Grüner S, et al. A critical role of platelet adhesion in the initiation of atherosclerotic lesion formation. J Exp Med 2002;196(07):887-896

43 Gazos-Lopes F, Oliveira MM, Hoelz LV, et al. Structural and functional analysis of a platelet-activating lysophosphatidylcholine of Trypanosoma cruzi. PLoS Negl Trop Dis 2014;8(08):e3077

44 Jy W, Mao WW, Horstman L, Tao J, Ahn YS. Platelet microparticles bind, activate and aggregate neutrophils in vitro. Blood Cells Mol Dis 1995;21(03):217-231

45 Gómez-Muñoz A, O’Brien L, Hundal R, Steinbrecher UP. Lysophosphatidylcholine stimulates phospholipase $\mathrm{D}$ activity in mouse peritoneal macrophages. J Lipid Res 1999;40(06):988-993 
46 Huang YH, Schäfer-Elinder L, Wu R, Claesson HE, Frostegård J. Lysophosphatidylcholine (LPC) induces proinflammatory cytokines by a platelet-activating factor (PAF) receptor-dependent mechanism. Clin Exp Immunol 1999;116(02):326-331

47 Badimon L, Suades R, Arderiu G, Peña E, Chiva-Blanch G, Padró T. Microvesicles in atherosclerosis and angiogenesis: from bench to bedside and reverse. Front Cardiovasc Med 2017;4:77

48 Hafiane A, Daskalopoulou SS. Extracellular vesicles characteristics and emerging roles in atherosclerotic cardiovascular disease. Metabolism 2018;85:213-222

49 Huber J, Vales A, Mitulovic G, et al. Oxidized membrane vesicles and blebs from apoptotic cells contain biologically active oxidized phospholipids that induce monocyte-endothelial interactions. Arterioscler Thromb Vasc Biol 2002;22(01):101-107

50 Hoefer IE, Steffens S, Ala-Korpela M, et al; ESC Working Group Atherosclerosis and Vascular Biology. Novel methodologies for biomarker discovery in atherosclerosis. Eur Heart J 2015;36(39): 2635-2642

51 Chatterjee M, Rath D, Schlotterbeck J, et al. Regulation of oxidized platelet lipidome: implications for coronary artery disease. Eur Heart J 2017;38(25):1993-2005

52 Tan KT, Lip GY. The potential role of platelet microparticles in atherosclerosis. Thromb Haemost 2005;94(03):488-492

53 Lavi S, McConnell JP, Rihal CS, et al. Local production of lipoprotein-associated phospholipase A2 and lysophosphatidylcholine in the coronary circulation: association with early coronary atherosclerosis and endothelial dysfunction in humans. Circulation 2007;115(21):2715-2721

54 Wilensky RL, Shi Y, Mohler ER III, et al. Inhibition of lipoproteinassociated phospholipase A2 reduces complex coronary atherosclerotic plaque development. Nat Med 2008;14(10):1059-1066

55 Mayr M, Grainger D, Mayr U, et al. Proteomics, metabolomics, and immunomics on microparticles derived from human atherosclerotic plaques. Circ Cardiovasc Genet 2009;2(04):379-388

56 Uchida Y. Recent advances in fluorescent angioscopy for molecular imaging of human atherosclerotic coronary plaque. J Atheroscler Thromb 2017;24(06):539-551
57 Ménégaut L, Masson D, Abello N, et al. Specific enrichment of 2-arachidonoyl-lysophosphatidylcholine in carotid atheroma plaque from type 2 diabetic patients. Atherosclerosis 2016;251: 339-347

58 Stegemann C, Drozdov I, Shalhoub J, et al. Comparative lipidomics profiling of human atherosclerotic plaques. Circ Cardiovasc Genet 2011;4(03):232-242

59 Gonçalves I, Edsfeldt A, Ko NY, et al. Evidence supporting a key role of Lp-PLA2-generated lysophosphatidylcholine in human atherosclerotic plaque inflammation. Arterioscler Thromb Vasc Biol 2012;32(06):1505-1512

60 Mannheim D, Herrmann J, Versari D, et al. Enhanced expression of LP-PLA2 and lysophosphatidylcholine in symptomatic carotid atherosclerotic plaques. Stroke 2008;39(05):1448-1455

61 Herrmann J, Mannheim D, Wohlert C, et al. Expression of lipoprotein-associated phospholipase $\mathrm{A}(2)$ in carotid artery plaques predicts long-term cardiac outcome. Eur Heart J 2009;30(23):2930-2938

62 Nishi K, Itabe $\mathrm{H}$, Uno M, et al. Oxidized LDL in carotid plaques and plasma associates with plaque instability. Arterioscler Thromb Vasc Biol 2002;22(10):1649-1654

63 Qin X, Qiu C, Zhao L. Lysophosphatidylcholine perpetuates macrophage polarization toward classically activated phenotype in inflammation. Cell Immunol 2014;289(1-2):185-190

64 Peter C, Waibel M, Radu CG, et al. Migration to apoptotic "find-me" signals is mediated via the phagocyte receptor G2A. J Biol Chem 2008;283(09):5296-5305

65 Kraemer BF, Borst O, Gehring EM, et al. PI3 kinase-dependent stimulation of platelet migration by stromal cell-derived factor 1 (SDF-1). J Mol Med (Berl) 2010;88(12):1277-1288

66 Stafford MJ, Watson SP, Pears CJ. PKD: a new protein kinase Cdependent pathway in platelets. Blood 2003;101(04):1392-1399

67 Prokazova NV, Zvezdina ND, Korotaeva AA. Effect of lysophosphatidylcholine on transmembrane signal transduction. Biochemistry (Mosc) 1998;63(01):31-37

68 Schmidt EM, Münzer P, Borst O, et al. Ion channels in the regulation of platelet migration. Biochem Biophys Res Commun 2011;415(01):54-60 